\title{
Electron optical benches for in-line and branched systems. A new bench designed for mirror-based aberration correction and low energy electron microscopy
}

\author{
Walter P. Skoczylas and Gertrude F. Rempfer \\ Department of Physics, Portland State University, Portland, Oregon 97207 \\ O. Hayes Griffith \\ Institute of Molecular Biology and Department of Chemistry, University of Oregon, Eugene, Oregon 97403
}

(Received 20 May 1994; accepted for publication 1 July 1994)

\begin{abstract}
A review of electron optical bench literature is presented, and the designs of two optical benches used by the authors are described. One bench was designed for testing individual electrostatic electron lenses and in-line optical systems, for example, emission electron microscopes and transmission electron microscopes. It has been in operation for many years. The second electron optical bench is new. It is a branched system designed for several purposes: to study correction of spherical and chromatic aberration with an electron mirror, and to gain experience with low energy electron microscopy (LEEM) optics. The alignment of the electron optical support structure is independent of the vacuum housing, and the bench is designed to be operated either horizontally or vertically. As a demonstration of the performance of the new bench in the horizontal mode, a test pattern on a silicon surface was imaged with LEEM optics.
\end{abstract}

\section{INTRODUCTION}

Electron optical benches play an important role in measuring the focal properties of electrostatic and magnetic lenses and their aberration coefficients. An optical bench is often the instrument of choice for testing the performance of prototype microscopes because of its versatility and adjustability. Whereas optical benches for visible light and glass lenses are readily available from commercial sources, electron optical benches must be designed and built individually by the research laboratories needing them. The main ways in which electron optical benches differ from light optical benches are that electrical connections must be made to the electron source and to each lens, and the entire optical system must be enclosed in a vacuum chamber. Additional factors such as translating the components without breaking vacuum, introducing magnetic shielding without affecting mechanical alignment, and viewing the image must be considered. The alignment of components in an electron optical bench is especially important for high magnification and low-energy beam applications. Surprisingly few electron optical benches are described in the literature. These publications are often difficult to locate, and no reviews on this subject have been published. We have three motivations for writing this paper. Our first reason is to provide a brief review of electron optical benches. The second reason is to describe the electron optical bench we have used in our laboratory for many years. The third purpose is to describe the design and operation of a new electron optical bench suitable for branched electron optical systems such as the optics required for aberration correction by means of an electron mirror, and for low energy electron microscopy. It is our hope that this gathering of the literature in one place and a discussion of our experiences with optical benches will make it easier for others entering the small but important field of experimental electron optics.

\section{REVIEW OF ELECTRON OPTICAL BENCHES}

Historically, the properties of electric and magnetic fields were first studied in sealed glass tubes, an outgrowth of early work on Geissler's or Braun's tubes and cathode-ray oscillographs. As magnetic and electrostatic lenses were developed in the 1930s and rudimentary electron microscopes assembled, the need to vary the position and combination of components made the construction of electron optical benches an important adjunct in electron optical research. In 1932 Brüche and Johannson at the Research Institute of the AEG in Berlin constructed their thermionic emission microscope using electrostatic lenses and obtained the first micrographs of heated metal and metal oxide cathodes. ${ }^{1,2}$ Brüche and Johannson aligned their electron optical components on a triangular rail, and then placed the entire assembly in a glass tube, sealed the ends, and evacuated it. At about the same time Knoll, Houtermans, and Schulze at the Berlin Technische Hochschule constructed an emission microscope using two magnetic lenses. ${ }^{3}$ The components of this microscope were also positioned on a triangular optical bench but the vacuum system was confined to a glass tube of smaller diameter held above the bench, with the magnetic coils on the outside. Both of these systems appear to be constructed using light-optical bench support systems. Systems similar to these have been set up in many other laboratories to study electron optics and to demonstrate the principles of electrostatic and magnetic lenses. There are naturally variations that did not use a triangular rail. For example, in 1938 Nicoll $^{4}$ described the focusing properties of an electron mirror formed between two cylinders in a glass tube. At about the same time, Klemperer and Wright made a thorough theoretical and experimental study of the focal properties and aberrations of two-cylinder lenses in a glass tube. ${ }^{5}$ They used a pepper-box type of diaphragm with two rings of holes to provide different radii of incidence. A rotary motion feedthrough permitted blockage of either the smaller or 


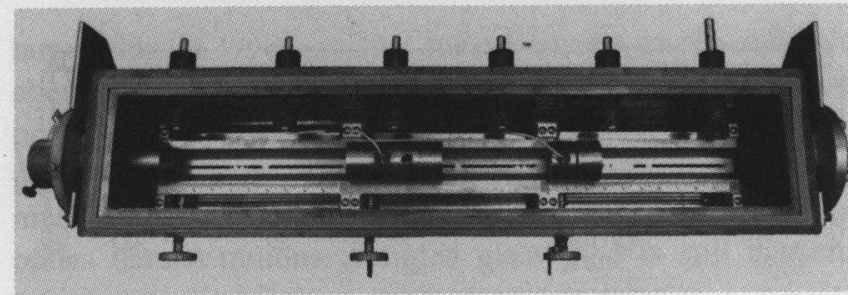

FIG. 2. Photograph of the Tektronix electron optical bench. Top view wit the glass plate cover removed. Three $V$-way sections placed end to end for a continuous channel down the middle of the bench. The
and aligns the electron lenses and other optical components. An electron gur is mounted on the left end of the bench and a phosphor-coated fiber optics
window is on the opposite end. In this photograph, the bench is set up for window is on the opposite end. In this photograph, the bench is set up for
test of an electrostatic objective lens. The electron optical path length from

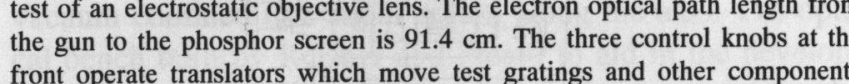
front operate translators which move test gratings and other component
along the $\mathrm{V}$ way. Six electrical feedthroughs are at the back. The mu-meta
magnetic shields which normally surround the beam during operation have magnetic shields which no
been removed for clarity.

insure that the optical axes of the components are aligned on diameter of the housings which we use is $53.95(+0,-0.02) \mathrm{mm}$

Each V-way section incorporates a linear translato which provides axial movement along the $\mathrm{V}$ way. Translatio is accomplished by turning a worm gear mounted under eac $\mathrm{V}$ way. The worm gear draws a carriage forward or backward in a slot in the bottom of the $\mathrm{V}$ way. This motion can be ransmitted to a housing on the $V$ way by a pin connecting the housing to the carriage. Three control knobs at the fron of the bench actuate the translators. Each knob is attached to a shaft, which is sealed through the vacuum wall of the bench with an $\mathrm{O}$ ring. The shaft connects to a pair of beve gears through an alignment coupler. (The coupler compensates for any axial or angular misalignment between the control shaft and the bevel gears. It also serves as a simp disengagement mechanism so the $V$ way can be remove from the bench without removing the control shaft/knob assembly.) The bevel gears transmit rotations of the contro knobs through $90^{\circ}$ to the worm gears.

There are six $2.22 \mathrm{~cm}$ diameter high-voltage feedthroug ports on the back of the bench for connections to the lenses and other components. An insulator with a metal rod throug he center serves as the high-voltage feedthrough. The insuator, either Rexolite (cross-linked polystyrene) or glazed ceramic, is $9.5 \mathrm{~cm}$ long and is sealed against the vacuum housing with an $\mathrm{O}$ ring. The high voltage rod is sealed to the insulator by an $\mathrm{O}$ ring between the outer end of the insulator and a shoulder on the rod. The assembly is held to the hou , and compression is applied to the O rings, by a nut on the vacuum end of the rod inside the chamber. The nut supported off the inner wall of the chamber by a secon insulator.

Flanged ports with $7.62 \mathrm{~cm}$ inside diameters are locate at each end of the bench. The ports can be used for mountin an electron gun, fiber-optics window, in-vacuum camera, additional electrical connections needed for a given expermen

The electron bench is set up for electrostatic optics, but in principle it could be used also for magnetic optics. The

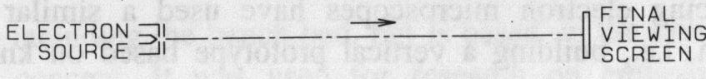

(a)

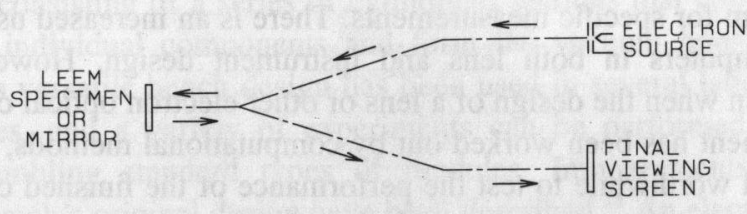

(b) FIG. 3. (a) A single axis arrangement used in the majority of electron optical
systems. (b) A branched electron optical path, as required in LEEM and

optical axis of an electrostatic lens coincides with the geometrical axis if the geometry is rotationally symmetric. This is not necessarily so for magnetic lenses because of possible asymmetries in the pole-piece material. Thus, while it is not necessary to provide $x$ and $y$ adjustments or beam steering for electrostatic lenses, if the lenses have been accurately machined and aligned, it may be necessary for magnetic lenses. A $z$-axis translation (along the $\mathrm{V}$ way) is provided as described earlier in this section. This mechanism has served mainly for moving phosphor screens, mechanical shutters and gratings and meshes used in lens tests.

The electron source for the bench experiments is the exit pupil of a thermionic triode gun with a thoriated-tungsten filament. The control electrode, or grid, is biased close to cutoff (independently of the emission current) for optimum beam characteristics. The filament and grid are supported off-ground on an insulating flange (Rexolite) inside the gun housing. The gun flange is sealed to one of the bench en langes with an $\mathrm{O}$ ring. The anode, at ground potential, is supported on the near end of the $V$ way inside the bench. $A$ gimbal-like arrangement enables $x$ and $y$ adjustments of the filament relative to the grid, and a threaded knob provides a $z$ adjustment. Sliding $x$ and $y$ adjustments of the grid and filament assembly relative to the anode are provided by two pairs of opposing screws acting on the gun flange. Thes various adjustments can be made during operation.

A $5 \mathrm{~cm}$ diameter pumping port on the bottom of the bench connects via a short coupling to a Veeco model VS-9 vacuum pumping station. The bench is supported on top of the pumping station by three adjustable feet. The vacuum system uses a trapped oil-diffusion pump backed by a mechanical pump. The vacuum inside the bench during an experiment is modest, typically $10^{-5}$ Torr $\left(1.3 \times 10^{-3} \mathrm{~Pa}\right)$ when the cold trap is used and $5 \times 10^{-5}$ Torr $\left(6.7 \times 10^{-3} \mathrm{~Pa}\right)$ without it.

Up to this point we have been discussing benches for in-line optical systems, all of which have a single axis, as illustrated in Fig. 3(a). However, in low-energy electron microscopy (LEEM) or in aberration correction with a mirror, both an incident and a returning beam are present, which necessitates a branched system. The incident and returning beams share a single axis in the LEEM/Mirfor branch, but

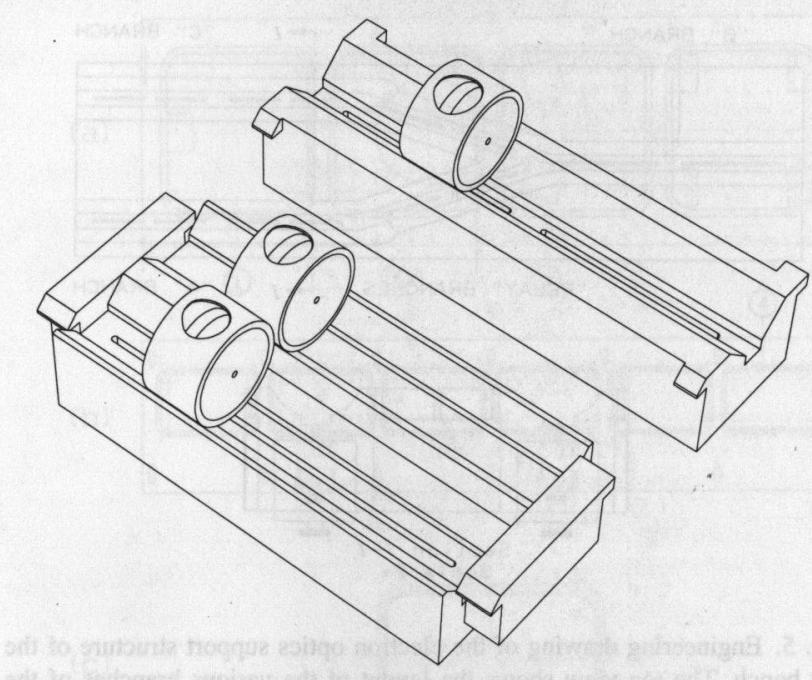

FIG. 4. Perspective drawings of the $\mathrm{V}$-way sections used in the modified Tektronix electron optical bench. Top: a single $\mathrm{V}$ way, the standard device
for holding electron lenses in the bench. Bottom: a double $\mathrm{V}$ way constructed for the mirror electron optics and beam separating system. Both
types of $\mathrm{V}$ ways are shown holding general purpose three electrode electrotypes of $\mathrm{V}$ ways are shown holding general purpose three electrode electio-
static leesses. The openings in the lens housings provide access for the hig oltage leads which contact the rims of the center electrodes. The cen telal.

up with the electron gun and the final viewing screen, which are necessarily on separate axes for independent control of the beams.

Recently, one of the two Tektronix-type benches at Portland State University was modified for studies of aberration correction by an electron mirror, and for preliminary tests of the optics for LEEM. The branching pattern used for separating the incident and returning beams is shown in Fig. 3(b). The separation is accomplished by a transverse magnetic field located on the LEEM/mirror axis where the inciden and returning beam axes diverge. Two other magnetic fields, each acting on one of the separated beams, produce deflections which bring the incident and returning axes into parallelism with the LEEM/mirror axis, but displaced from it in opposite directions. To avoid impairment of resolution by deflection aberrations the optics are adjusted so that deflections take place at image planes. Relay lenses transfer the images between deflectors without change of magnificatio or orientation. A photograph of the modified bench, set up for the mirror studies, has been published elsewhere. ${ }^{13}$

In order to support the optics for the separated beams we replaced the $\mathrm{V}$-way section at the right end of the bench with one having two tracks (double $V$ way). Figure 4 shows a single $\mathbf{V}$ way (upper) and a double $\mathbf{V}$ way (lower). Electrostatic lenses of the three-electrode unipotential type are shown on the $V$ ways. The openings in the lens housings are for high-voltage connectors which contact the rims of the center electrodes. The center electrodes are insulated by means of Rexolite spacers from the end electrodes, which are at ground potential. The center-to-center distance betwee the double $\mathrm{V}$-way tracks is $5.7 \mathrm{~cm}$. It was convenient to have the incident beam on the rear track, and the returning bea on the front track, as indicated schematically by the top an bottom tracks of Fig. 3(b). A Y-shaped module, housing the deflectors and relay lenses, was constructed to transfer the way fouble $V$ way the single $V$ way on the left and to transfer the returning beam from the single $\mathrm{V}$ way to the front track of the double $\mathrm{V}$ way. In order for the relay optics to fit into the space available, the relay lenses were made to half scale. The accelerating voltage was correspondingly reduced to $15 \mathrm{kV}$ from the normal operating voltage of $30-40 \mathrm{kV}$ for full-sized lenses.

The foregoing changes in the electron optical arrangement necessitated a modification in the vacuum housing to enable viewing and recording of the experimental results. The flanged port at the right end of the bench was cut away, and the area machined to accept a rectangular adapter having an off-set window in line with the front axis of the double $V$ way. While it would have been desirable to mount an electron gun on this adapter, in line with the rear axis, there was insufficient space. Instead, a smaller gun lacking the desire adjustability during operation was placed directly on the rear $\checkmark$ way.

Several other limitations, besides inadequate space in the bench, added to the need for an improved design. There is no provision for moving the magnets to adjust the center of deflection during operation, or for adjusting the gun with respect to the optical axis. It is difficult to adequately shiel the beam from magnetic fields because the shields for the lenses can extend only as far down as the contact between the lens housings and the $V$ way. The number of high-voltage feedthroughs is insufficient for some of the experiments, and pump-down time is unduly long $(30-40 \mathrm{~min})$.

The most compelling reason for a new design has to do with alignment of the electron beam. While the bench is entirely adequate for the original purpose of measuring lens focal properties and aberrations, the alignment requirements for matching and canceling aberrations in the mirror studies, and achieving good resolution in the LEEM studies, are much more stringent. Misalignment problems in the mirro and LEEM studies in the old bench are due mainly to the following shortcomings: (i) The electron optical components are supported on three separate $\mathrm{V}$-way sections, which in turn are aligned by locating surfaces in the vacuum housing. Not only is it more difficult to achieve alignment in the first place, but also this arrangement leaves the alignment vulnerable to small distortions of the housing occurring during pump down. (ii) The lack of adjustability of the gun and the magnets during operation, noted above, makes the alignmen process a time consuming and tedious series of approximations. (iii) The practical result of the magnetic shielding problem, also noted above, is that shielding is imperfect, In spo con the bean misaligne

In spite of the shortcomings described above, this optical bench recently produced the first LEEM and mirror electron microscopy (MEM) images of biological specimens s $^{34}$ and in use for deter. in use for deterin optical setups.

\section{THE NEW ELECTRON OPTICAL BENCH}

The design of the new bench began with the support structure for the branched electron optics. Our approach was 
to design the support structure first ${ }_{2}$ and then decide on the vacuum housing to accommodate it. The branching pattern is essentially the same as in the old bench [Fig. 3(b)], withou the constraint of limited space.

\section{A. The support structure}

The branches of the support structure are called by different names depending on the mode of operation of the electron optical system. To avoid confusion in discussing the relation between the branches we will refer to the incident, or condenser, branch simply as the $\mathrm{C}$ branch, the mirror branch or LEEM branch as the $\mathrm{B}$ branch, and the returning branch as the $\mathrm{A}$ branch. There is no confusion in referring to the relay branches. The ideal conditions on the support structure which we are aiming at are listed below.

(1) The beam axes in the various branches should lie in the same plane.

(2) The B axis and the two relay axes should meet at a point. (3) The relay axes should make equal and opposite angle with the $B$ axis

(4) The A and C axes should be parallel to the B axis, an displaced from it by equal amounts in opposite directions.

(5) The separation of the A and C branches should be large enough to permit full-sized relay lenses to be used in the relay branches.

(6) The support system should allow magnetic shields to completely encircle the electron optical components. The support system should also allow for axial adjustments of various components (e.g., deflecting magnets, screens, meshes, etc.) during operation.

After considering alternatives (in particular, a system of lens tubes) we decided that the alignment conditions could be satisfied best by a trough system milled out of a single block of metal. In this way the alignment of the various branches of the system would not depend on support from the vacuum housing. A fringe benefit of the trough choice is the relative ease of access to the electron optics for modifcations and servicing as contrasted with accomplishing these same tasks in a branched system of lens tubes. The troug profile in the new structure is a circular arc rather than a $V$ way. In effect the alignment control is the same as in a le tube. The circular profile is better than $\mathrm{a} \mathrm{V}$ profile or a pair of rods, in that an undersized component cannot move as far out of line.

A drawing of the top view of the support structure, fabricated out of aluminum, is shown in Fig. 5 (upper). The center-to-center separation of the $\mathrm{A}$ and $\mathrm{C}$ troughs is 15.24 $\mathrm{cm}$. The angle between the relay axes and the C, B, and axes is $17.5^{\circ}$. A section view of the trough is shown in Fig. 5 (lower). The centerline of the trough arc is $1.14 \mathrm{~cm}$ above the surface of the support structure. The radius of the arc larger $(3.02 \mathrm{~cm})$ than the radii of the electron optical housings $(2.7 \mathrm{~cm})$. The housings are spaced off the troughs by precision rings at the ends of the housings, thus allowing for mu-metal shields to extend completely around the electro lenses and other components. The mu metal serves to shiel against both electric and magnetic fields. Slots in the botto
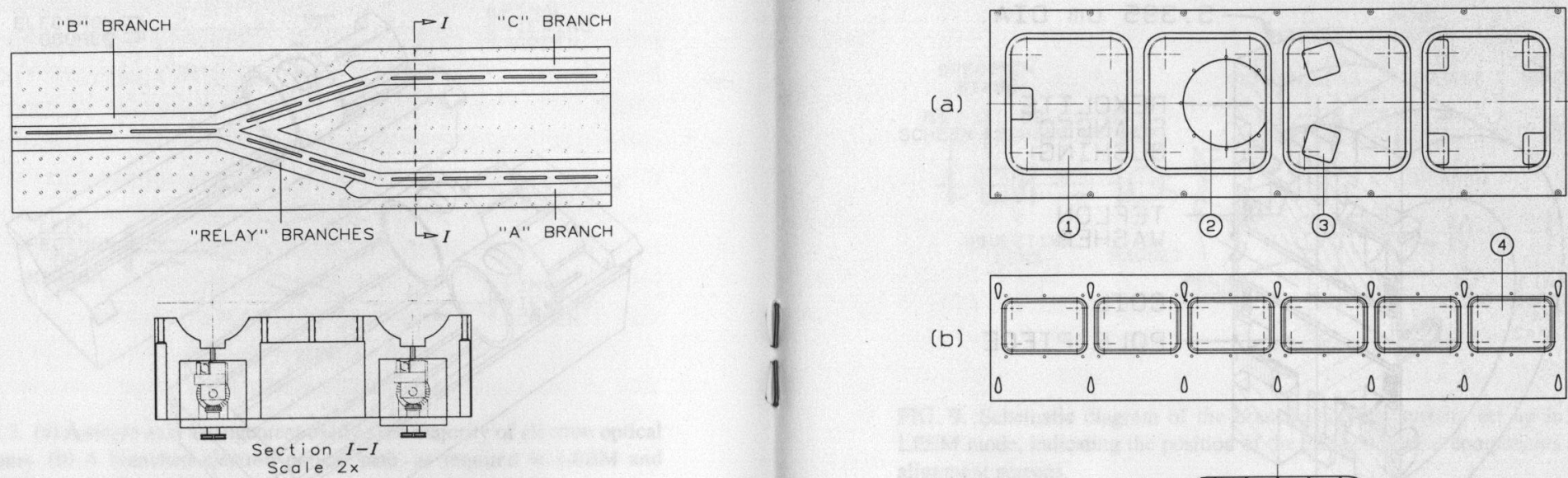
FIG. 5. Engineering drawing of the electron optics support structure of the
new bench. The top view shows the layout of the various branches of the
electron optical system. Also shown are numerous threaded holes through the top of the structure which are useful for clamping components and other apparatus in place. The electron optical elements are supported and aligned along the beam branches by troughs machined into the support structure. A profile of the troughs can be seen in the $2 X$ scale section view below. The view. The linear translators connect to components along the beam axes by means of slots cut through the bottom of the troughs. There are two trans lators in the $\mathrm{A}$ branch, two in the $\mathrm{C}$ branch, one in each of the relay
branches, and one in the $B$ branch. The support structure is machined out a solid block of in and $7.6 \mathrm{~cm}$ high. The radius of the trough arc is $3.02 \mathrm{~cm}$.

of the troughs allow for translation of components by mecha-

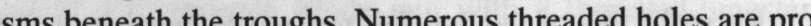
vided in the block for mounting various fixtures and accessories. These threaded holes also enable components to be clamped in place for alternative operation in a vertical orientation. The first phase of the experiments is being done with the trough system in a horizontal orientation. However, fo high-resolution tests of LEEM and corrected optics a vertica orientation is preferred in order to minimize effects of me-
ofing chanical vibrations. A vibration isolation platform will also be used to minimize vibrations.

\section{B. The vacuum housing}

The simplest approach to construction of the vacuum housing was to start with a length of rectangular steel tubing having dimensions large enough to easily accommodate the electron optics and allow for the versatility needed in exploring several modes of operation. A $91.4 \mathrm{~cm}$ length of $20.3 \mathrm{~cm}$ by $30.4 \mathrm{~cm}$ tubing with a wall thickness of $1.27 \mathrm{~cm}$ was chosen to serve as the mainstay of the vacuum housing. Figure 6 shows engineering drawings of the top view (a), side view (b), and end view (c) of the housing after machining. O-ring grooves were machined into the end surfaces of the tube [Fig. 6(c)], and threaded holes were provided for fastening on steel end plates. The end plates are interchangeable and serve either as blanks or as mounts for electron guns, output windows, or an in-vacuum camera. Six large rectangular ports were machined on the front and back sides of the housing [Fig. 6(b)]. O-ring grooves matching the shape an size of the ports were cut around the perimeter of each port for sealing. Blind holes were drilled and tapped into the out-

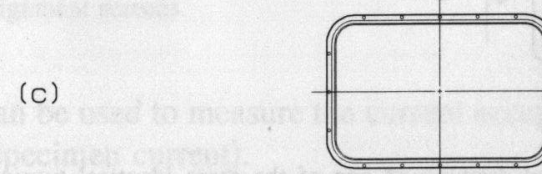

FIG. 6. Engineering drawings of the vacuum housing of the new electron viewing ports, (2) pumping port, (3) $z$-axis linear translator ports, and (4) side ports. The housing was fabricated from a length of rectangular stef ports were machined into the housing. Blind holes in the housing have been drilled and tapped for fastening on the port cover plates. To prevent corrosion, the housing and steel port cover plates were electroless nickel plated The dimensions of the housing are
high. The wall thickness is $1.3 \mathrm{~cm}$.

side of the housing for fastening on interchangeable steel cover plates carrying an array of feedthroughs or acte ste depending on the experiments being performed. Ports in the dhe housing were machined in a simila way. However, because of the large radius of the rounded way. However, because of the large radius of the rounded edges of the steel tube, the additional step of counterboring
was used in machining the tapped blind holes. The bottom of was used in machining the tapped blind holes. The bottom of the counterbore provides a flat surface parallel to the top and
bottom surfaces of the housing, which makes the tapped holes more convenient to use. [In the side view of the housing, Fig. 6(b), these holes appear as "tear-drop" shaped patterns.] On the top side of the housing there are four large ports for access to and viewing of the experiments [Fig. $6(\mathrm{a})]$. These ports are sealed with $1.9 \mathrm{~cm}$ thick hinged glas plates. The seven $5.08 \mathrm{~cm}$ square ports [Fig. 6(a)] machine into the bottom side of the vacuum housing are for rotary feedthroughs which operate the $z$-axis translators under the troughs in the support structure. The $15.2 \mathrm{~cm}$ diameter round port on the bottom of the housing connects to an oil-pumped vacuum system. Seals are made with Viton 0 rings. Operating pressures are in the low $10^{-5}$ Torr $\left(10^{-3} \mathrm{~Pa}\right)$ range without a cold trap, and in the low $10^{-6}$ Torr $\left(10^{-4} \mathrm{~Pa}\right)$ range with a cold trap. These pressures are adequate for the electron optics experiments we are currently doing. Figure shows a perspective drawing of the vacuum housing with the support structure in place.

This design using large area ports with the O-ring sealing grooves built into the vacuum housing provides the flexibility needed for our electron optics experiments. The type, number, and location of feedthroughs can easily be changed

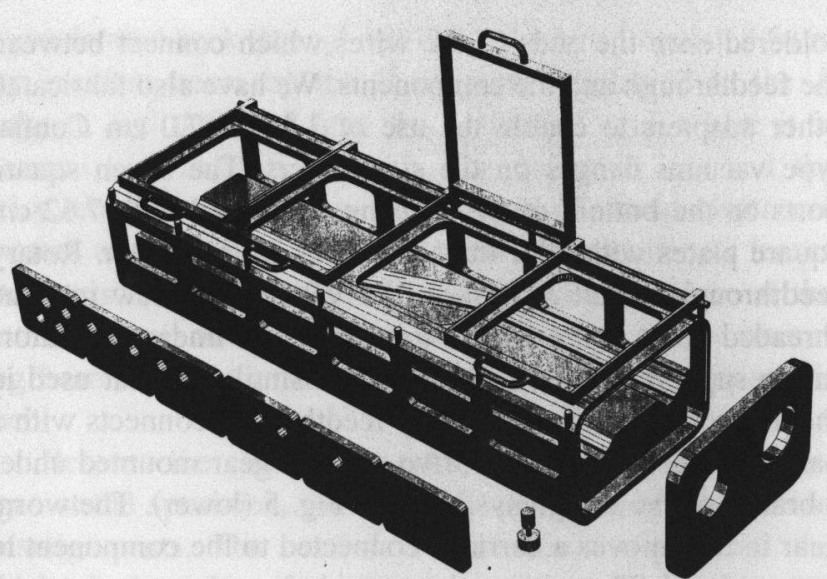

FIG. 7. Perspective drawing of the vactum housing of the new bench with the electron optics support structure in place. In the foreground is a selection One of seven $z$-axis translator control knobs is displayed at the bottom of open position.

to fit the arrangement of components in the bench by merely changing the side, end, and top cover plates. Only one face of the cover plate needs to have a good smooth sealing surface, which makes the plates easy to fabricate. We have stanfeedthrough ports. This allows the use of standard baseplate fittings and feedthroughs on the system. Some of the various fittings and feedthroughs on the system. Some of the various ground of Fig. 7. When feedthroughs, such as the highvoltage and multiple pin low-voltage feedthroughs, were not available in the styles we needed, we designed and built our own. Our noncommercial high-voltage feedthrough design is similar to that used in the Tektronix bench. A metal rod, bored at each end to accept a mini-banana plug, serves as the high-voltage lead. The rod fits through an opening on the axis of a Rexolite insulator. The insulator is $7.62 \mathrm{~cm}$ long, and has a diameter of $3.81 \mathrm{~cm}$ which is stepped down to 2.54 $\mathrm{cm}$ to fit the opening in a side cover plate. An $\mathrm{O}$ ring in a groove in the bore inside the insulator seals to the rod, and an $\mathrm{O}$ ring in a groove on the outside of the insulator seals to the wall of the opening in a cover plate. The low-voltage feedthrough uses a commercial eight-pin electrical feedthrough (Insulator Seal, Inc. P/N 9411027). This device comes with a $1.905 \mathrm{~cm}$ long by $1.905 \mathrm{~cm}$ diameter sleeve surrounding eight $1.27 \mathrm{~mm}$ diameter pins. We use a quick disconnect baseplate adapter, similar to the MDC Corp. P/N 650005 , to seal the low-voltage feedthrough to the $2.54 \mathrm{~cm}$ diameter hole in the side cover plate. On the outside of the vacuum housing, we designed an adapter to consect the pins of the commercial feedthrough to banana jacks. This enables the use of common laboratory cables to make connections between the feedthrough and the various power supplies required to run the bench. On the inside of the vacuum housing, where connectors on the components are much smaller than banana plugs, we designed an adapter to convert the pins of the original commercial feedthrough to $1.02 \mathrm{~mm} \mathrm{di-}$ ameter sockets (Hypertronics Corp. P/N YSK0102-004AH). Mating pins (Hypertronics Corp. P/N YPN0102-004G) are

Electron-optic benches $\quad 3189$ 

gear in turn moves a carriage connected to the component to be translated. The vacuum housing, being of magnetic steel, provides some protection against magnetic fields. However, we expect to line the housing with one or more layers of mu metal to further reduce the field inside the chamber and to shield against any permanent magnetism which might be present in the housing. Openings in the shielding will be provided to enable viewing of the alignment screens.

\section{EXPERIMENTAL TESTS IN THE NEW ELECTRON
OPTICAL BENCH}

Two electron guns were built to serve as electron sources for the experiments. The guns are modeled after the gun built in the early 1970s for the Elektros Inc. transmission electron microscope. The Elektros gun is a triode gun with a thoriated tungsten filament, and is fully adjustable during operation. The electron lenses are electrostatic.
both the LEEM and the correction experiments is the same both the LEEM and the correction experiments is the same as the new objective lens for the photoelectron microscope
(PEM or PEEM) at the University of Oregon. The PEM lens has a swept-back design to allow for direct illumination of has a swept-back design to allow for direct illumination of
the specimen when used in the PEM mode. ${ }^{35}$ The relay lenses also are swept-back lenses. The swept-back design lenses also are swept-back lenses. The swept-back design
enables us to use a relatively small angle of $17.5^{\circ}$ between enables us to use a relatively small angle of $17.5^{\circ}$ between
the LEEM/mirror axis and the relay axes. This is the smallest angle which accommodates the relay lenses. The other lenses in the experiments are general purpose three-electrode unipotential lenses. An accelerating voltage of $20 \mathrm{kV}$ is used in the experiments. Lens voltages are obtained from a voltage divider. The four relay lenses are designed to operate at the same voltage. Leads from the inside ends of the high-voltage feedthroughs contact the rims of the center electrodes through openings in the lens housings and the mu-metal shields.

The deflecting magnets are electromagnetic. A section view is shown in Fig. 8. The magnetizing current is supplied through the low-voltage feedthroughs. The pole pieces are circular with a diameter of $2.54 \mathrm{~cm}$. They are made of steel with mu-metal disks of the same diameter fastened to their faces. The purpose of the mu-metal disks is to short out any small lateral fields which might be caused by nonuniformity in the steel pole-piece material. This is a temporary measure and the $\mu \mathrm{m}$-metal disks will be removed when the steel pole pieces are replaced by pole pieces of soft iron. There are 250 turns of $37 \mathrm{AWG}$ magnet wire on each pole piece. A current of about $75 \mathrm{~mA}$ is used to deffect the $20 \mathrm{kV}$ beam through the required $17.5^{\circ}$ angle. The pole pieces are isolated elec-

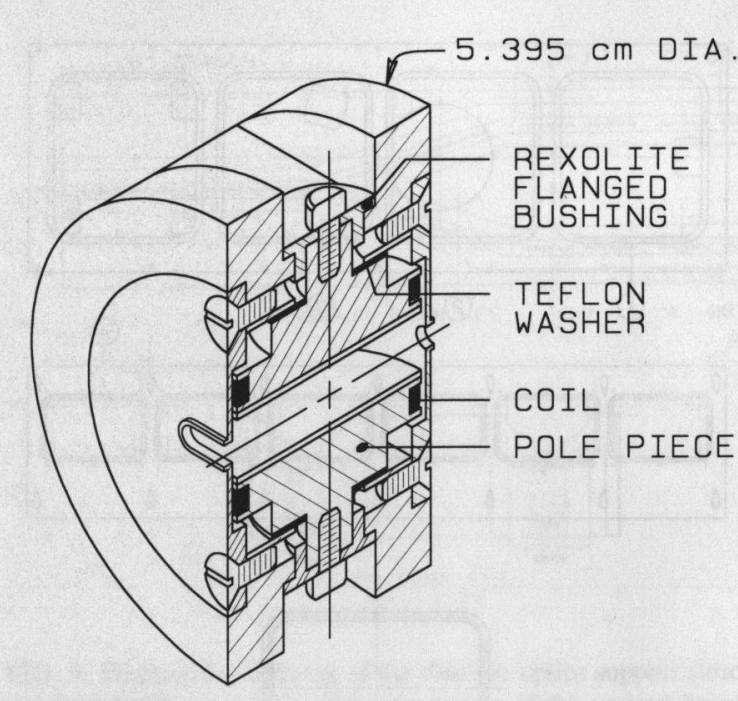

FIG. 8. Isometric sectional drawing of one of the three identical turning magnets. The two steel pole pieces of the electromagnet are circular with
diameters of $2.54 \mathrm{~cm}$, and are faced with mu-metal disks of the same diameter. The mu-metal disks were included as a precautionary measure to sho
out any lateral fields which might be generated by inhomogeneities in the out any lateral fields which might be generated by inhomogeneities in the
steel pole-piece material. There are 250 turns of 37 AWG magnet wire
wound onto each pole picec. A current of about $75 \mathrm{~mA}$ is required to deffece wound onto each pole piece. A current of about $75 \mathrm{~mA}$ is required to defect
the $20 \mathrm{kV}$ beam through the $17.5^{\circ}$ angle used in the beam separating syster of the new bench. The pole pieces are isolated electrically from the extern pole pieces from the external circuit, and a flanged Rexolite bushing insuates the screw which secures the pole piece to the external circuit. The pol pieces can be used as electrodes for making small vertical deffections of the electron beam. A positive DC voltage (relative to ground) is applied to on
pole piece and an equal negative DC voltage to the other. The gap between pole piece and an equal negative $\mathrm{DC} v \mathrm{v}$

trically from the external magnetic circuit and can be used as electrodes for making small vertical deflections of the electron beam. The pole piece faces are separated by a gap of $6.35 \mathrm{~mm}$. The return path for the magnetic field has the same outside diameter as the lens housings, $5.395 \mathrm{~cm}$. The magnets are supported on extensions of the A, B, and C troughs at the intersections with the relay axes. Axial adjustments of their positions can be made during operation by means of the translators under the troughs. An $x, y, z$ specimen translato for the LEEM mode has not yet been completed. However, temporary manipulator mounted on one of the front cover plates enables the specimen to be translated in one dimensio at right angles to the optical axis (vertically).

A single power supply (CPS model 100N) provides hig voltage to the electron gun and the voltage divider. An offground noncommercial supply provides the heating curre for the gun filament. The grid bias is obtained from a potentiometer either across a battery or in the high voltage line in series with the voltage divider. Low-voltage power supplies Hewlett-Packard model E3611A) provide the magnetizing currents for the deflectors. For aberration correction experiments the mirror electrode is maintained at up to $5 \mathrm{kV}$ negaive of the cathode by a variable $0-5 \mathrm{kV}$ power supply (Beran Associates model PMT-50A/N) operated off-ground. he LEEM mode the specimen voltage is supplied from the cathode of the electron gun via a small variable bias-voltage supply in the high-voltage line. A nanometer circuit in the high-voltage line between the specimen and the bias supply
SPECIMEN
STAGE

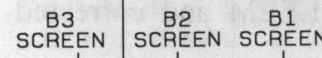

$+\square 01=10$

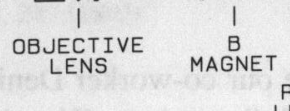

MACNET STEEAMER LENS

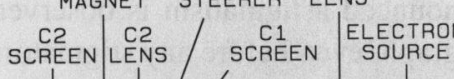
$-\theta=0-\frac{1}{1}=0-\mathbb{E}$ RELAY
LENSES MAGNET STEAM G AAMERA

FIG. 9. Schematic diagram of the branched optical system, set up in the alignment screens.

can be used to measure the current accepted by the specimen (specimen current).

Our first experiments on the new bench have been done in the LEEM mode. The LEEM studies, aside from being of interest in their own right, are valuable in arriving at the alignment conditions needed for the aberration-correction experiments. Furthermore, the LEEM studies enable us to test the beam separator alone without the correcting mirror. A schematic diagram of the optical arrangement for LEEM is shown in Fig. 9, and a photograph of the bench set up for LEEM is shown in Fig. 10. (For other LEEM optics designs see Bauer, Liebl and Senftinger, and Tromp and Reuter. The incident beam originats at the electron gun at the upper ight in Figs. 9 and 10 . The bean travels toward the left along the condenser branch to the $\mathrm{C}$ magnet at the flected onto the relay axis. The relay lenses wed of the without change of magnification or orientalion to the B magnet, whese bes is de wean passes though secimen After interting with the specimen the bem is

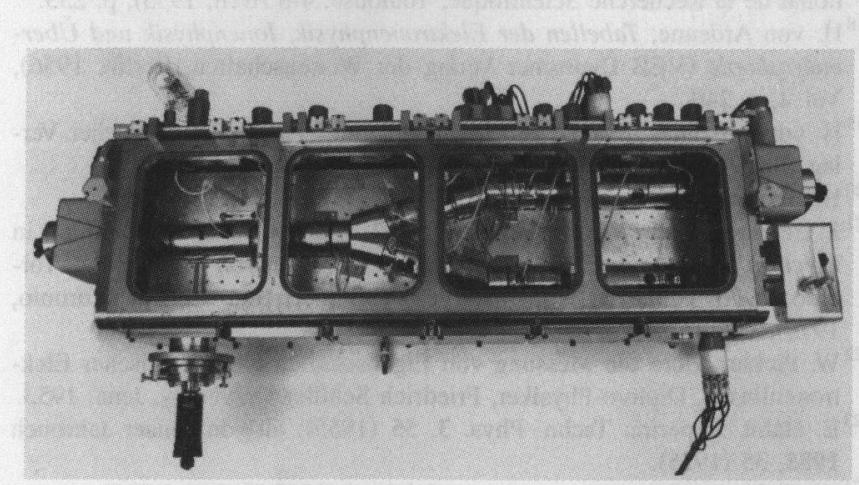

FIG. 10. Photograph of the new electron optical bench. This is a top view

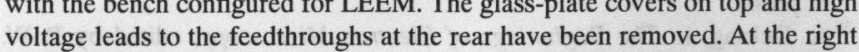
front is a typical multipin (8) low voltage $(<1000 \mathrm{~V})$ electrical feedthrough. Three other low-voltage feedthroughs can be seen at the rear. The specimen manipulator control is at the left front. A vacuum compatible internal roll
film camera is on the right end of the bench toward the front. Just to the rear of the camera is the electron gun for the system. An identicial electron gun is shown at the left end of the bench. The left end gun position is used when the bench is operated in a IEM mode, or for trouble shoorige reaccelerated back through the objective lens, separated from the incident beam by the B magnet, transferred to the A magnet, and deflected onto the projection axis, where it is focused to a magnified image. The objective lens and the relay lenses are focused so that images of the specimen are located at the centers of the magnets. The condenser lenses are focused to form an image of the electron source at the entrance pupil of the objective lens (exit pupil for the returning beam) so that a parallel beam is incident on the specimen.

Because the electrons are decelerated to such low voltages at the specimen, the LEEM image is very sensitive to misalignments. The controls on the electron gun and the axial translators for the deflecting magnets (both adjustable during operation) which have been incorporated in the new bench, have greatly facilitated beam alignment. Stray magnetic fields have been reduced to an acceptable level for our present tests by two layers of mu-metal shielding surrounding the electron beam. The machining of the trough and the electron optical components also is sufficiently precise for our initial tests. However, the LEEM image is especially sensitive to misalignment of the objective lens. We plan to incorporate a means for making fine adjustments of the objective lens transverse to the axis in order to correct for the small errors which are inevitable, even in parts machined to high tolerances. Furthermore, the diameter of the field of view at the specimen is very small (of the order of $40 \mu \mathrm{m}$ in diameter or less), and its image formed by the objective stage at the B magnet and transferred to the A magnet is still very small. Hence, it is necessary to have the first projector lens (P1) lined up well with this image if one is to see any part of the final image on the viewing screen. In order to optimize the alignment we plan to provide a means for fine adjustments of P1 as well as the objective lens. We want to emphasize the importance of having viewing screens at strategic locations along the optical path (e.g., as in Fig. 9). Phosphorcoated discs with central openings are easy to include, and they are crucial in simplifying the task of aligning the beam. A LEEM micrograph of a test specimen is shown in Fig. 11. The specimen was obtained from Ted Pella, Inc. It is usually used for magnification and distortion calibration in SEM. The calibration pattern was fabricated by electronbeam lithography on a single-crystal silicon wafer. The squares have a period of $9.9 \mu \mathrm{m}$. The lines are $1.9 \mu \mathrm{m}$ wide. This micrograph was taken with the specimen biased $6 \mathrm{~V}$ positive of the cathode of the electron gun. The image was recorded without stigmators or an aperture stop. The electron image was recorded directly on film with an in-vacuum rollfilm camera modified for this purpose. The electronic magnification was $600 \times$, and the exposure time was $2 \mathrm{~s}$. The pressure in the system was $3 \times 10^{-5}$ Torr $\left(4 \times 10^{-3} \mathrm{~Pa}\right)$. The ccelerating voltage was $20 \mathrm{kV}$.

The magnification of the objective stage is about $7 \times$. The two projection lenses together can multiply this magnification up to about $2500 \times$ at the camera, or output window. The image at the output window can be viewed on a video monitor at up to $16000 \times$. It is interesting to observe the topography. Almost imperceptible adjustments of the beam 


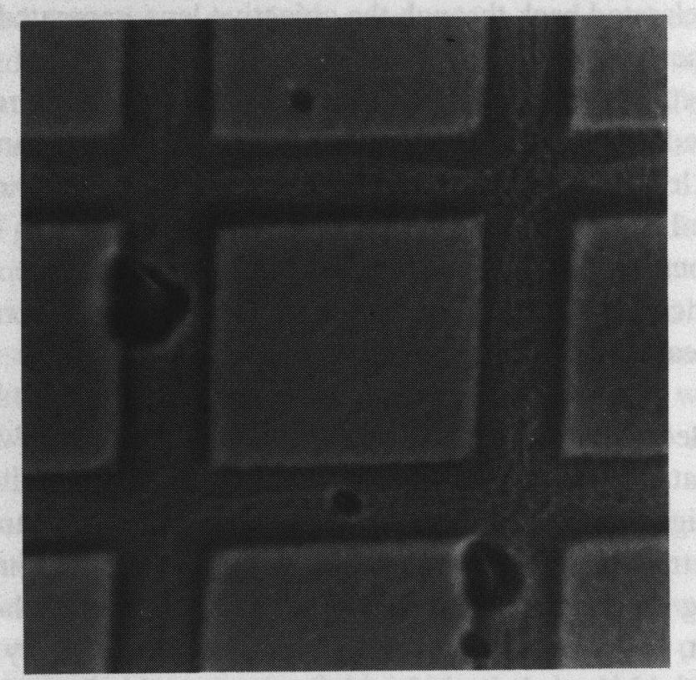

FIG. 11. Micrograph of a magnification test specimen recorded with the beam lithography on a single-crystal silicon wafer. The squares have a period of $9.9 \mu \mathrm{m}$, and the lines are $1.9 \mu \mathrm{m}$ wide. This micrograph was taken with the specimen biased $6 \mathrm{~V}$ positive of the electron gun cathode. The image was recorded without stigmators or an aperture stop at an electronic means of the in-vacuum camera shown in Fig. 10 at the right-hand end of the vacuum bench. The exposure time was $2 \mathrm{~s}$ on IIford 50 photographic film. The pressure in

steerer in the condenser branch can move the shadows cas by topographic details from side to side. As in PEM, the specimen is the negative electrode of an electric field, an specimen topography creates microfields at the specimen surface. The deflecting power of the microfields for the low energy electrons entances contrast in the image. This effect is advantageous for fine detail. However, only small to moderate specimen topography, where deffections are not too large to be included in the imaging beam, can be imaged well.

Optimizing LEEM resolution will require a number of improvements, including a fully functional specimen manipulator, an aperture stop, sigmators, and fine-focusing capability. Also needed will be addicinal shielding to protec against both $\mathrm{DC}$ and $\mathrm{AC}$ magnetic fields, vibration isolation, and finalized deflecting magnets using soft iron or other high-permeability material. At present he magnetic shielding is marginal, but with the addition of magnetic shields lining the vacuum chamber, it will be substantially improved. The locations of the feedthroughs, viewing ports, and other openings into the vacuum system have been established, and the shields can be made with only small gaps to accommodate these openings. Stringent pre-conditions must also be satisfied for high-resolution tests of an aberration-corrected PEM or other instrument. ${ }^{39}$ One pre-condition is identifying and eliminating spurious sources of small misalignments. A second pre-condition is the demonstration that the beam separating system is optimal. The availability of the new electron optical bench will greatly facilitate studies with an electron mirror leading to the experimental demonstration of aberranounced astigmatism is observed in this preliminary micrograph, even before any stigmators are in pduced, is evidence that the beam separatio optics is working well.

\section{ACKNOWLEDGMENTS}

We are pleased to acknowledge our co-worker Denis M. Desloge for valuable assistance and discussions. We wish to thank the following people who contributed information about electron optical benches and the development of electron microscopes: Dr. A. W. Agar, Dr. A. B. Bok, Dr. P. E. Bovey, Dr. A. Delong, Dr. W. Engel, Dr. M. Felsmann. Dr. O. Fortagne, Dr. F. Hasselbach, Dr. P. W. Hawkes, Dr. L. Y. Huang, Dr. P. Kruit, Dr. T. Mulvey, Dr. R. O. Partsch, Dr. R. W. Rempfer, Dr. K.-H. Schmidt, Dr. A. Septier, Dr. E.-A. Soa, and Dr. K. Yagi. We also acknowledge the expert support services provided by the Science Shops at Portland State University and the University of Oregon. This work was supported by Grant BIR-8907619 from the National Science Foundation.

${ }^{1} \mathrm{E}$. Brüche and H. Johannson, Naturwissenschaften 20, 353 (1932).

${ }^{2}$ E. Briche and H. Johannson, Naturwissenschaften 20, 353 (1932).
${ }^{2}$. Büche and H. Johannson, Ann. Phys. 15, 145 (1932).

${ }^{3}$ M. Knoll, F. G. Houtermans, and W. Schulze, Z. Phys. 78,340 (1932). ${ }^{4}$ F. H. Nicoll, Proc. Phys. Soc. London 50, 891 (1938)

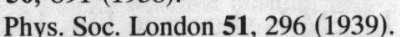
${ }^{7}$ C. Fert, J. Phys. Radium 13, 64A (1952).

J. H. Reisner and R. G. Picard, Rev. Sci. Instrum. 19, 556 (1948).

${ }^{9}$ E. B. Bas, Z. Angew. Phys. 6, $404(1954)$

"I M. S. Mauck, Master's Thesis, Portland State University, 1969

${ }^{12}$ G. F. Rempfer, J. Appl. Phys. 57, 2385 (1985).

${ }^{13}$ G. F. Rempfer and M. S. Mauck, Optik 92, 3 (1992).

${ }^{14}$ M. S. Mauck, Ph.D. Thesis, Portland State University, 1993.
${ }^{15}$ L. Marton, M. M. Morgan, D. C. Schubert, J. R. Shah, and J. A. Simpson,

J. Res. Natt. Bur. Stand. 47, 461 (1951).
16.

${ }^{16} \mathrm{E}$. Ruska, Z. wiss. Mikrosk. mikrosk. Tech. 60, 317 (1952).
${ }^{17} \mathrm{E}$. Ruska, Les Techniques Recentes en Microscopie Electronique et Cor${ }^{17}$ E. Ruska, Les Techniques Recentes en Microscopie Electronique et Cor-
pusculaire (Comptes Rendus de Colloques Internationaux du Centre Napusculaire (Comples Rendus de Coulloques Interrationaux du Centre Na-
tional de la Recherche Scientifique, Toullouse, $4-8$ Avril, 1955), p. 253. ${ }^{18} \mathrm{H}$. von Ardenne, Tabellen der Elektronenphysik, Ionenphysik und Über-
mikroskopie (VEB Deutscher Verlag der Wissenschaften, Berlin, 1956), Vol. I, p. 240.

Wer Werne, Tabellen zur angewandten Physik (VEB Deutscher Verlag der Wissenschaften, Berlin, 1964), Vol. II, p. 672

${ }^{21} \mathrm{~J}$. Geiger, H. Schmoranzer, H. Jakobs, H. Katterwe, and B. Schröder, in

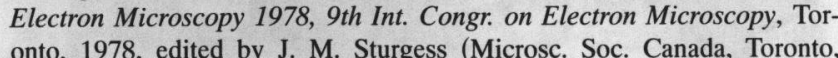
1978), Vol. 1, p. 4.

${ }^{22}$ W. Fickler, Uuber die Messung von Eigenschaften elektrostatischer Elektronenlinsen, Diplom-Physiker, Friedrich Schiller University, Jena, 1953.
23. ${ }^{23}$ E. Hahn, Expe

${ }^{24}$ W. Fickler and E. Guyenot, Jenaer Jahrbuch 1963 II, 345 (1963). ${ }^{25}$ A. W. Agar, in The Growth of Electron Microscopy, edited by T. Mulvey

in press).
${ }^{26}$ A. Delong, in The Beginnings of Electron Microscopy, edited by P. W.
Hawkes, Adv. Electron. Electron Phys. Suppl. 16 (Academic, New York, 1985), p. 66 .
${ }^{27}$ P. Duncumb,

phia, Peumb, in Proc. 5 th Int. Congr. for Electron Microscopy, PhiladelNew York, 1962), Vol. 1, p. KK-4.

${ }^{28} 8$ A. Septier, C. R. Acadd. Sci. Paris 245,1406 (1957).
${ }^{29}$ P. Hawkes, Ph.D. Dissertation, University of Cambridge, 1963.
${ }^{30} \mathrm{P}$. Grivet, Electron Optics (Pergamon, Oxford, 1965). ${ }^{32}$ F. Hassselbach, Z. Phys. B 71, 443 (1988). (1993). K. F. J. Heinrich, in Electron Probe Quantitation edited by K K F. J. Heinrich and D. E Newbury (Plenum, New York, 1991) p. 317. O. H. Griffith, K. K. Hedberg, D. Desloge, and G. F. Rempfer, J. Microsc 168. $249(1992)$
${ }^{35}$ G. F. Rempfer, W. P. Skoczylas, and O. H. Griffith, Ultramicroscopy 36, 196 (1991).

${ }^{36} \mathrm{E}$. Bauer, Ultramicroscopy 17, 51 (1985)

3. Liebl and B. Sentinger, Ultramicroscopy 36, 91 (1991).

${ }^{39}$ W. P. Stoczlos, G. F. Remfer, 252 (1991). 


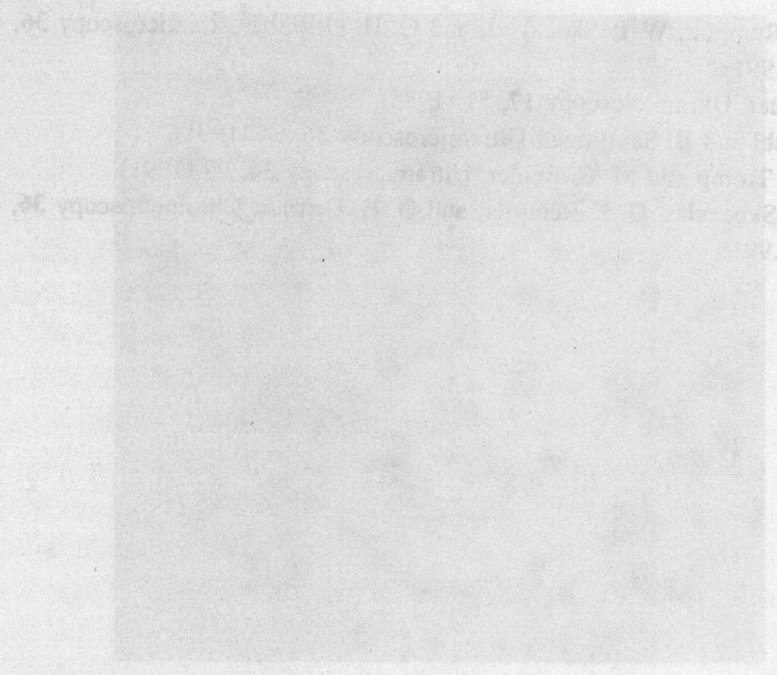

actes gat

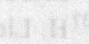

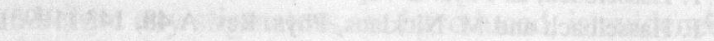

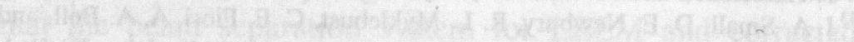

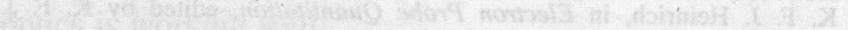
and

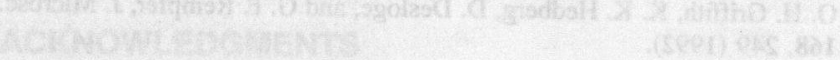
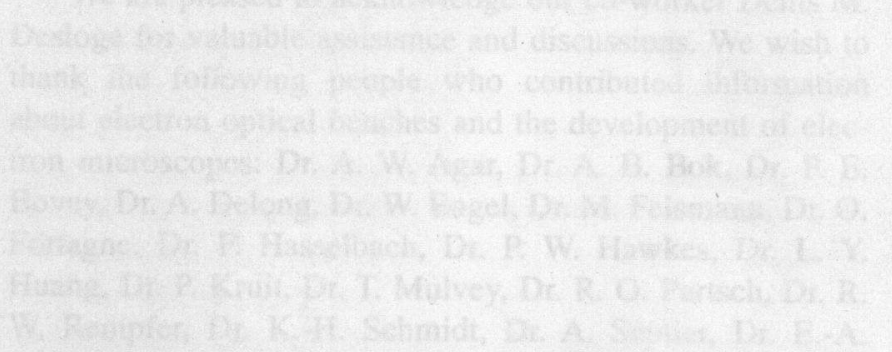

(⿻)

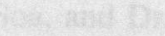

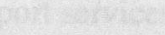

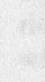

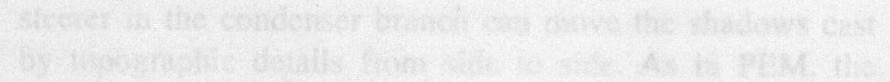

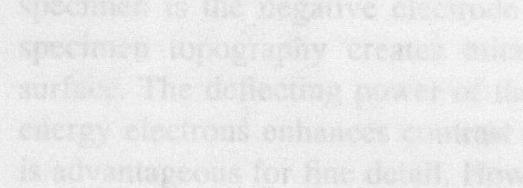

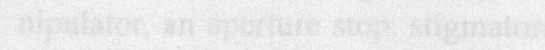

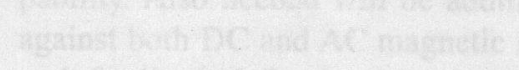

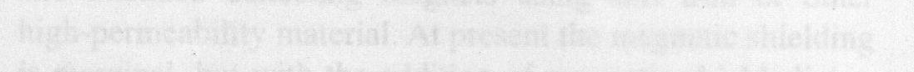

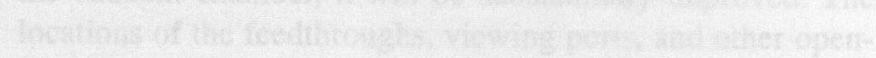

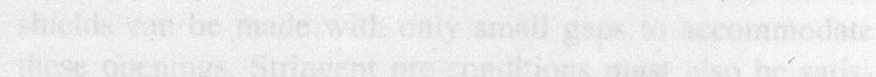

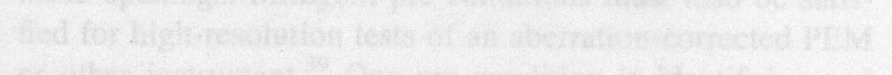

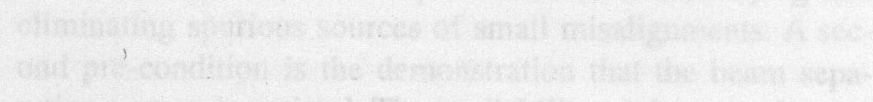

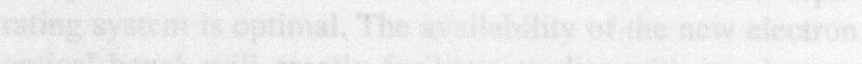

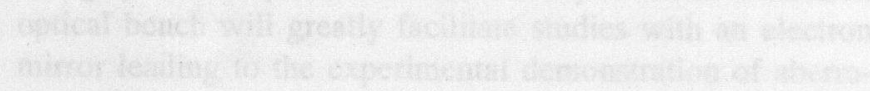

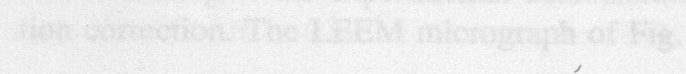

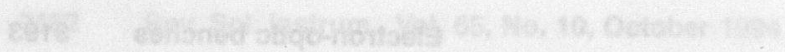

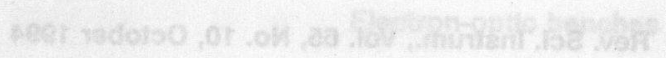

